\title{
Verification Theorems for HJB equations
}

\section{Mauro Garavello*}

Dipartimento di Matematica e Applicazioni, Università di Milano Bicocca

Via R. Cozzi 53 - Edificio U5

20125 Milano (Italy)

E-mail: mauro.garavello@unimib.it

We study an optimal control problem in Bolza form and we consider the value function associated to this problem. We prove two verification theorems which ensure that, if a function $W$ satisfies some suitable weak continuity assumptions and a Hamilton-Jacobi-Bellman inequality outside a rectifiable set of codimension one, then it coincides with the value function.

Control Systems: Theory, Numerics and Applications

30 March - 1 April 2005

Rome

${ }^{*}$ Speaker. 


\section{Introduction}

In this paper we consider a control system of the type:

$$
\dot{x}=f(t, x, u), \quad u \in U
$$

where $x \in \mathbb{R}^{n}$ is the state, $U \subset \mathbb{R}^{q}$ is the control space and $f$ is the controlled dynamic. Given a target $S \subset \mathbb{R}^{n}$, a running cost $L(t, x, u)$, a fi nal cost $\psi(t, x)$ and an initial condition $\left(\hbar, x_{0}\right)$, we consider the optimal control problem in Bolza form. We defi ne in the usual way the value function $V\left(t_{0}, x_{0}\right)$ to be the infi mum of the problem with initial condition $\left(\hbar, x_{0}\right)$. It is well known that, under suitable conditions, $V$ satisfi es the Hamilton-Jacobi-Bellman equation in viscosity sense [1] and it is possible to isolate $V$ as the unique solution. The proof is based on the dynamic programming principle.

Therefore given a function $W$, it is possible to determine if $W$ coincide with the value function, checking if it is a viscosity solution to the HJB equation. This type of theorems, called verifi cation theorems, are useful, for example, when a candidate value function is produced by means of the construction of a synthesis [13]. It is then natural to ask for minimal conditions under which a function $W$ coincides with the value function. If we know that $W$ was obtained via a synthesis then the inequality $W \geq V$ is granted by construction, thus we take this assumption. Then, for $W$ to coincide with the value function, we prove it is suffi cient that, outside a rectifi able set of codimension one, both $W$ is differentiable and it satisfi es a Hamilton-Jacobi-Bellman inequality. Moreover, we make use of only some weak continuity assumptions, already used in [13] to prove optimality of a regular extremal synthesis, see Theorem 1 and Theorem 2 for details. A fi rst result in this direction can be found in [2], where the HJB inequality is asked outside a countable collection of Lipschitz continuous manifolds of positive codimension. Notice that, for an optimal control problem, the value function is indeed differentiable outside a closed rectifi able set of codimension one, see [他.

We start considering the main assumptions for the problem and presenting two technical lemmas, one of which dealing with the cardinality of the intersections between admissible trajectories and a rectifi able set.

The fi rst case we treat is the problem of fi nite time. We defi ne a value function as the infi mum, over all admissible trajectories reaching the target in fi nite time. The main result of this part is Theorem 11 which permits to verify if the function $W$ coincides with the value function. In particular we need the differentiability of $W$ outside a rectifi able set and the fact that $W$ must satisfy a HJB differential inequality in the same set.

Next, we consider the infi nite time problem. In this case the value function 5.1) is defi ned as the infi mum of the cost functional over all admissible trajectories reaching the target in infi nite time. The main result of this section is Theorem 1 which gives suffi cient conditions on the function $W$ to ensure the equality between $W$ and the value function. In this case, we consider a suitable neighborhood $S_{1}$ of the target $S$ and we suppose that the fi nal cost $\psi$ is defi ned on $S$ in order to give sense to the limit in the defi nition of the value function [5.1). As a corollary of Theorem 1 and Theorem 2 we can treat a mixed case (see also [12]), considering at the same time the trajectories reaching the target both in fi nite time and in infi nite time. 
A key ingredient for Theorem 1 and Theorem 2 is the positiveness of the Lagrangian $L$, in order to prevent some bad phenomena such as the permanence of the system for an arbitrary interval of times in a region where $L$ is negative making the value function equal to $-\infty$.

\section{Acknowledgements}

The author wishes to thank Prof. B. Piccoli for having proposed him the study of this problem and for his useful advice.

\section{Preliminaries}

We consider a control system:

$$
\dot{x}(t)=f(t, x(t), u(t)), \quad(t, x) \in \Omega, \quad u(t) \in U
$$

where

(A-1) $\Omega$ is an open and connected subset of $\mathbb{R} \times \mathbb{R}^{n}$.

(A-2) $U$ is a non-empty subset of $\mathbb{R}^{q}$, for some $q \geq 1, q \in \mathbb{N}$.

(A-3) $\mathcal{U}=L^{p}(\mathbb{R} ; U)$ with $1 \leq p<+\infty$ is the set of admissible controls.

(A-4) $f: \Omega \times U \rightarrow \mathbb{R}^{n}$ is measurable in $t$, continuous in $(x, u)$, differentiable in $x$ and, for each $u \in U$, $D_{x} f(\cdot, \cdot, u)$ is bounded on compact sets. Moreover there exists $\varphi_{1}: \mathbb{R} \rightarrow \mathbb{R}^{+}$integrable and for every $K$, compact subset of $\Omega$, there exist a modulus of continuity $\omega_{K}$ and a constant $L_{K}>0$ such that, if $(t, x) \in K$ and $(t, y) \in K$, then

$$
\left\{\begin{array}{l}
|f(t, x, u)-f(t, y, u)| \leq \omega_{K}(|x-y|) \\
(f(t, x, u)-f(t, y, u)) \cdot(x-y) \leq L_{K}|x-y|^{2} \\
|f(t, x, u)| \leq L_{K}\left(\varphi_{1}(t)+|u|^{p}\right) .
\end{array}\right.
$$

We consider a function $L: \Omega \times U \rightarrow \mathbb{R}$ and assume:

(A-5) $L$ is measurable in $t$ and continuous in $(x, u)$. Moreover, there exist $\varphi_{2}: \mathbb{R} \rightarrow \mathbb{R}^{+}$integrable and, for every $R \geq 0, C_{R} \geq 0$ such that

$$
|L(t, x, u)| \leq C_{R}\left(\varphi_{2}(t)+|u|^{p}\right), \quad|(t, x)| \leq R
$$

In this paper we indicate with $x\left(\cdot ; u, t_{0}, x_{0}\right)$ the solution to (2.1) such that $x\left(t_{0} ; u, t_{0}, x_{0}\right)=x_{0}$. Defi ne the value function:

$$
V\left(t_{0}, x_{0}\right):=\inf _{\substack{u \in \mathcal{U} \\\left(T, x\left(T ; u, t_{0}, x_{0}\right)\right) \in S}}\left\{\int_{t_{0}}^{T} L\left(s, x\left(s ; u, t_{0}, x_{0}\right), u(s)\right) d s+\psi\left(T, x\left(T ; u, t_{0}, x_{0}\right)\right)\right\}
$$

where $S$ - the target - is a closed subset of $\mathbb{R} \times \mathbb{R}^{n}$ contained in $\Omega, \psi: S \rightarrow \mathbb{R}$ is the fi nal cost. Now we need the following defi nitions (introduced in [13]): 
Definition 1. We call a function $W: \mathbb{R}^{n} \rightarrow \mathbb{R} \cup\{+\infty\}$ weakly upper semicontinuous (w.u.s.c.) at a point $x$ if $\liminf _{y \rightarrow x} \limsup _{z \rightarrow y} W(z) \leq W(x)$. Moreover $W$ is w.u.s.c. if it is w.u.s.c. at every point of $\mathbb{R}^{n}$.

Remark 1. A function $W: \mathbb{R}^{n} \rightarrow \mathbb{R} \cup\{+\infty\}$ is w.u.s.c. at $x$ if and only if, for any sequence $\delta_{j}>0$, $\delta_{j} \rightarrow 0$ there exist a sequence $x_{j} \rightarrow x$ and a sequence $\varepsilon_{j}>0$ such that

$$
\left|y-x_{j}\right| \leq \varepsilon_{j} \quad \Rightarrow \quad W(y) \leq W(x)+\delta_{j} .
$$

Note that we can choose $0<\varepsilon_{j} \leq \delta_{j}$.

Definition 2. Suppose that we have a time-varying Lipschitz-continuous vector fi eld $X$ on $\mathbb{R}^{n}$ and $W: \Omega \rightarrow \mathbb{R} \cup\{+\infty\}$. We say that $W$ has the no downward jumps property (NDJ) along $X$ if for any $[a, b] \ni t \mapsto \gamma(t)$, solution to $\dot{\gamma}(t)=X(t, \gamma(t))$ such that $(t, \gamma(t)) \in \Omega \forall t \in[a, b]$, we have $\liminf _{h \downarrow 0} W(t-h, \gamma(t-h)) \leq W(t, \gamma(t))$, whenever $\left.\left.t \in\right] a, b\right]$.

Definition 3. $A$ subset $A$ of $\Omega$ is an $n$-dimensional rectifi able set if there exist $A_{1}$ and $A_{2}$ such that $A=A_{1} \cup A_{2}, A_{1}$ is a fi nite or countable union of connected Lipschitz submanifolds of positive codimension, and $\mathcal{H}^{n}\left(A_{2}\right)=0$, where $\mathcal{H}^{k}$ is the k-dimensional Hausdorff measure.

\section{Two lemmas}

In this section we present two technical lemmas.

Lemma 1. Fix an element $\omega \in U, t^{\prime}<t^{\prime \prime}$ and $x \in \mathbb{R}^{n}$ with $\left(t^{\prime \prime}, x\right) \in \Omega$. Assume that there exists $\mathcal{W}$, an open neighborhood of $x$ in $\mathbb{R}^{n}$, such that $\zeta^{y}(\cdot)$, the solution to $\dot{\zeta}^{y}(t)=f\left(t, \zeta^{y}(t), \omega\right)$ with $\zeta^{y}\left(t^{\prime \prime}\right)=y$, is defined on $\left[t, t^{\prime \prime}\right]$ for any $y \in \mathcal{W}$ and $\left(t, \zeta^{y}(t)\right) \in \Omega \forall t \in\left[t^{\prime}, t^{\prime \prime}\right]$. Let $A$ be an $n$ dimensional rectifi able set.

Then for a.e. $y \in \mathcal{W}$ the set $B^{y}:=\left\{t \in\left[t^{\prime}, t^{\prime \prime}\right]:\left(t, \zeta^{y}(t)\right) \in A\right\}$ is fi nite or countable.

Proof. We can write $A=A_{1} \cup A_{2}$, where $A_{1}=\cup_{j} M_{j}$ and $\left\{M_{j}\right\}_{j \in J}$ is a fi nite or countable family of connected submanifolds of $\mathbb{R}^{n+1}$ of codimension $d_{j}>0$, and $\mathcal{H}^{n}\left(A_{2}\right)=0$. After replacing each $M_{j}$ by a fi nite or countable family of open submanifolds of $M_{j}$, we may assume that the $M_{j}$ are embedded. Defi ne $\widetilde{\mathcal{W}}:=] t^{\prime}, t^{\prime \prime}\left[\times \mathcal{W}\right.$ and let $\Phi$ be the map $\widetilde{\mathcal{W}} \ni(t, y) \mapsto\left(t, \zeta^{y}(t)\right) \in \Omega$. The Jacobian of $\Phi$ is

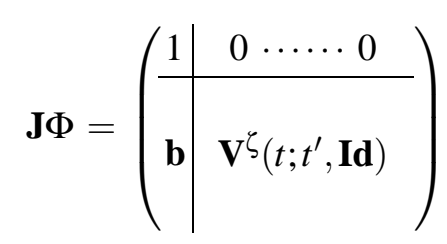

where $\mathbf{b}$ is the column vector $f\left(t, \zeta^{y}(t), \omega\right)$ and $\mathbf{V}^{\zeta}\left(t ; t^{\prime}, \mathbf{I d}\right)$ is the fundamental matrix solution to the linear system

$$
\dot{v}(t)=-D_{x} f\left(t, \zeta^{y}\left(-t+t^{\prime}+t^{\prime \prime}\right), w\right) \cdot v(t)
$$

such that $\mathbf{V}^{\zeta}\left(t^{\prime} ; t^{\prime}, \mathbf{I d}\right)=\mathbf{I d}$. So the determinant of $\mathbf{J} \Phi$ is equal to the determinant of $\mathbf{V}^{\zeta}\left(t ; t^{\prime}, \mathbf{I d}\right)$, which is equal to $\exp \int_{t^{\prime}}^{t} \operatorname{tr}\left(-D_{x} f\left(s, \zeta^{y}\left(-s+t^{\prime}+t^{\prime \prime}\right), \omega\right)\right) d s$, by Liouville's theorem (see [9]). In 
particular $\operatorname{det}(\mathbf{J} \Phi)$ is strictly positive for any $t \in\left[t^{\prime}, t^{\prime \prime}\right]$. Moreover, by (A 4$) \operatorname{tr}\left(-D_{x} f\right)$ is bounded on compact sets and then there exist $c>0, C>0$ such that $0<c \leq \operatorname{det}(\mathbf{J} \Phi) \leq C$.

So $\Phi$ is a Lipschitz diffeomorphism. In particular we have $\mathcal{H}^{n}\left(\Phi^{-1}\left(A_{2}\right)\right)=0$. Now, for each $j$, let us consider $\widetilde{M}_{j}:=\Phi^{-1}\left(M_{j}\right)$. It is a locally Lipschitz embedded submanifold of codimension $d_{j}>0$. So we may suppose that $\widetilde{M}_{j}$ is locally described as the image of a bi-Lipschitz continuous function $\varphi_{j}: U \rightarrow \mathbb{R}^{n+1}$, where $U$ is an open and bounded subset of $\mathbb{R}^{m}$, with $1 \leq m \leq n$.

First we suppose that $m<n$. We obviously have that $\mathcal{H}^{m}\left(\varphi_{j}(U)\right) \leq L \mathcal{H}^{m}(U)<+\infty$ where $L$ is the lipschitz constant for $\varphi_{j}$ and so (see [5]) $\mathcal{H}^{n}\left(\varphi_{j}(U)\right)=0$. This implies that $\mathcal{H}^{n}\left(\Pi\left(\widetilde{M}_{j}\right)\right)=0$. Since $\mathcal{L}^{n}$ coincides with $\mathcal{H}^{n}$ in $\mathbb{R}^{n}$ (see [巨]), we conclude that $\mathcal{L}^{n}\left(\Pi\left(\widetilde{M}_{j}\right)\right)=0$.

So we may suppose that $m=n$. We defi ne the set

$$
Z_{j}:=\left\{(t, y) \in \widetilde{M}_{j}: \varphi_{j} \text { not differentiable at } x=\varphi_{j}^{-1}(t, y)\right\} .
$$

By Rademaker theorem, we obtain that $\mathcal{H}^{n}\left(Z_{j}\right)=0$ and so $\mathcal{L}^{n}\left(\Pi\left(Z_{j}\right)\right)=0$. We now consider the function

$$
\Pi \circ \varphi_{j}: U \rightarrow \mathbb{R}^{n}
$$

the set

$$
S_{j}:=\left\{u \in U: \Pi \circ \varphi_{j} \text { differentiable at } u \text { and } D^{\prime}\left(\Pi \circ \varphi_{j}\right)(u) \text { not surjective }\right\}
$$

and the set

$$
Z_{j}^{(1)}:=\varphi_{j}\left(S_{j}\right)
$$

By Sard's lemma (see [7]), $\mathcal{L}^{n}\left(\Pi\left(Z_{j}^{(1)}\right)\right)=0$. So the set

$$
\mathcal{B}:=\Pi\left(\Phi^{-1}\left(A_{2}\right)\right) \cup\left(\bigcup_{j} \Pi\left(Z_{j}\right)\right) \cup\left(\bigcup_{j} \Pi\left(Z_{j}^{(1)}\right)\right)
$$

has Lebesgue measure 0 in $\mathbb{R}^{n}$.

Let $y \in \mathcal{W} \backslash \mathcal{B}$. Then $\left(t, \zeta^{y}(t)\right) \notin A_{2}$ if $t^{\prime}<t<t^{\prime \prime}$. To obtain the thesis, it is suffi cient to show that, for each $j$, the set $E_{j}=\{t \in] t^{\prime}, t^{\prime \prime}\left[:\left(t, \zeta^{y}(t)\right) \in M_{j}\right\}$ is at most countable. Fix $j$ and suppose $\bar{t} \in E_{j}$. Then $\left(\bar{t}, \zeta^{y}(\bar{t})\right) \in M_{j}$ and $(\bar{t}, y) \in \widetilde{M}_{j}$. By the fact that $y \notin \mathcal{B}$, we have $\frac{\partial}{\partial t} \notin T_{\widetilde{M}_{j}}(\bar{t}, y)$. This fact permits to conclude that $(t, y) \notin \widetilde{M}_{j}$ if $0<|t-\bar{t}|<\varepsilon$ for $\varepsilon$ suffi ciently small. Therefore $t$ is an isolated point of $E_{j}$ and the lemma is proved.

Lemma 2. Let $g$ be a real-valued function on a compact interval $[a, b]$. Assume that there exists a $f$ nite or countable subset $E$ of $[a, b]$ with the following properties:

(A-1) $\liminf _{h \downarrow 0} \frac{g(x+h)-g(x)}{h} \geq 0$ for all $x \in[a, b[\backslash E$,

$(A-2) \liminf _{h \downarrow 0} g(x+h) \geq g(x)$ for all $x \in[a, b[$,

$(A-3) \liminf _{h \downarrow 0} g(x-h) \leq g(x)$ for all $\left.\left.x \in\right] a, b\right]$.

Then $g(b) \geq g(a)$.

For a proof of this lemma see [13, Lemma B.1]. 


\section{Problem with fi nite time}

We indicate with $\partial Q$ the topological boundary of an arbitrary $Q \subseteq \mathbb{R} \times \mathbb{R}^{n}$.

Theorem 1. Suppose (A-Z)-(A-5) hold. Let $Q \subseteq \Omega$ be an open subset containing $S$. Let $W: \bar{Q} \rightarrow \mathbb{R}$ be a lower semicontinuous function verifying the NDJ property along every time-varying vector fi eld of the type $f(t, x, u)$ with $u \in U$ fixed. Moreover we assume that, for each $t, W(t, \cdot)$ is w.u.s.c. and that:

i) $W \geq V$.

ii) $W \leq \psi$ on $S$.

iii) At every point $(t, x) \in \partial Q$ one has

$$
W(t, x)=\sup _{(s, y) \in Q} W(s, y)
$$

iv) There exists an n-dimensional rectifi able set set $A \subseteq \Omega$ such that $W$ is differentiable on $Q \backslash A$ and satisfi es

$$
W_{s}(s, y)+\inf _{\omega \in U}\left\{W_{y}(s, y) \cdot f(s, y, \omega)+L(s, y, \omega)\right\} \geq 0 \quad \text { on } Q \backslash A .
$$

v) $L \geq 0$.

Then $W=V$ on $Q$. If $Q=\Omega$ we can drop hypotheses iii) and $Q$.

Proof. We have to prove that $W \leq V$. Suppose by contradiction that there exists $\left(t_{0}, x_{0}\right) \in Q$ such that $W\left(t_{0}, x_{0}\right)>V\left(t_{0}, x_{0}\right)$. Then there exists $M \in \mathbb{R}$ such that $W\left(t_{0}, x_{0}\right)>M>V\left(t_{0}, x_{0}\right)$. So we can fi nd $\varepsilon>0, \delta>0$ such that

$$
V\left(t_{0}, x_{0}\right)+\varepsilon \leq M-\varepsilon
$$

and, by the lower semicontinuity of $W$,

$$
\left|x-x_{0}\right|<\delta \Rightarrow W\left(t_{0}, x\right)>V\left(t_{0}, x_{0}\right)+\varepsilon .
$$

We can fi nd $u^{*} \in \mathcal{U}$ such that $x^{*}(\cdot):=x\left(\cdot ; u^{*}, t_{0}, x_{0}\right)$ satisfi es $\left(T, x^{*}(T)\right) \in S$ and

$$
\int_{t_{0}}^{T} L\left(s, x^{*}(s), u^{*}(s)\right) d s+\psi\left(T, x^{*}(T)\right) \leq V\left(t_{0}, x_{0}\right)+\frac{\varepsilon}{2} .
$$

Moreover, by [3, Théorèm IV.9], there exist $h \in L^{p}\left(\left[t_{0}, T\right]\right)$ and, for every $\eta>0, u^{\sharp}=u^{\sharp}(\eta) \in \mathfrak{u}$ such that $\left\|u^{\sharp}-u^{*}\right\|_{L^{p}\left(\left[t_{0}, T\right]\right)} \leq \eta, u^{\sharp}$ piecewise constant, left continuous and $\left|u^{\sharp}\right| \leq h$ a.e. Hence, if we denote by $x^{\sharp}(\cdot)$ the trajectory $x\left(\cdot ; u^{\sharp}, T, x^{*}(T)\right)$, for $\eta$ suffi ciently small, we have

$$
\left|\int_{t_{0}}^{T}\left[L\left(s, x^{\sharp}(s), u^{\sharp}(s)\right)-L\left(s, x^{*}(s), u^{*}(s)\right)\right] d s\right| \leq \frac{\varepsilon}{2}
$$


and

$$
\left|x^{\sharp}(t)-x^{*}(t)\right|<\frac{\delta}{2} \quad \forall t \in\left[t_{0}, T\right] .
$$

Fix an interval $\left.] t^{\prime}, t^{\prime \prime}\right]$ such that $u^{\sharp}(t) \equiv \omega$ in $\left.] t^{\prime}, t^{\prime \prime}\right]$. Suppose that $\left(t, x^{\sharp}(t)\right) \in Q \forall t \in\left[t^{\prime}, t^{\prime \prime}\right]$. Let $\zeta^{y}(t)$ be the trajectory associated to the constant control $\omega$ and such that $\zeta^{y}\left(t^{\prime \prime}\right)=y$. By the fact that $d\left(\partial Q,\left\{\left(t, x^{\sharp}(t)\right): t \in\left[t^{\prime}, t^{\prime \prime}\right]\right\}\right)>0$, we can fi nd an open neighborhood $\mathcal{W}$ of $x^{\sharp}\left(t^{\prime \prime}\right)$ in $\mathbb{R}^{n}$ such that $\left(t^{\prime \prime}, y\right) \in Q \forall y \in \mathcal{W}$ and $\left\{\left(t, \zeta^{y}(t)\right): t \in\left[t^{\prime}, t^{\prime \prime}\right]\right\} \subseteq Q \forall y \in \mathcal{W}$. By Lemma 11, we have that for a.e. $y \in \mathcal{W}$ the set $B^{y}:=\left\{t \in\left[t^{\prime}, t^{\prime \prime}\right]:\left(t, \zeta^{y}(t)\right) \in A\right\}$ is at most countable.

Since $W$ is w.u.s.c. for every fi xed $t$, then for every $\delta_{j} \rightarrow 0, \delta_{j}>0$ there exist $x_{j} \rightarrow x^{\sharp}\left(t^{\prime \prime}\right)$, and $0<\varepsilon_{j} \leq \delta_{j}$ such that

$$
\left|x_{j}-y\right| \leq \varepsilon_{j} \quad \Rightarrow \quad W\left(t^{\prime \prime}, y\right) \leq W\left(t^{\prime \prime}, x^{\sharp}\left(t^{\prime \prime}\right)\right)+\delta_{j} .
$$

For $j$ suffi ciently big, we can find $y,\left|y_{j}-x_{j}\right| \leq \varepsilon_{j}$, such that $B^{y_{j}}$ is at most countable. Consider the following function defi ned on $\left[t, t^{\prime \prime}\right]$ :

$$
\varphi_{j}(t):=W\left(t, \zeta^{y_{j}}(t)\right)+\int_{t^{\prime}}^{t} L\left(s, \zeta^{y_{j}}(s), \omega\right) d s
$$

By the choice of $y_{j}$ and the hypotheses $-v$ ) , $\varphi_{j}$ is differentiable a.e. with a nonnegative derivative. By the lower semicontinuity of $W$ and the NDJ condition, it follows that $\varphi_{j}$ verifi es the hypotheses of Lemma且 and so $\varphi_{j}\left(t^{\prime}\right) \leq \varphi_{j}\left(t^{\prime \prime}\right)$. Thus

$$
W\left(t^{\prime}, \zeta^{y_{j}}\left(t^{\prime}\right)\right) \leq W\left(t^{\prime \prime}, \zeta^{y_{j}}\left(t^{\prime \prime}\right)\right)+\int_{t^{\prime}}^{t^{\prime \prime}} L\left(s, \zeta^{y_{j}}(s), \omega\right) d s .
$$

Now, using (4.6) and the fact that $\zeta^{y_{j}}\left(t^{\prime \prime}\right)=y_{j}$ we obtain

$$
W\left(t^{\prime}, \zeta^{y_{j}}\left(t^{\prime}\right)\right) \leq W\left(t^{\prime \prime}, x^{\sharp}\left(t^{\prime \prime}\right)\right)+\delta_{j}+\int_{t^{\prime}}^{t^{\prime \prime}} L\left(s, \zeta^{y_{j}}(s), \omega\right) d s .
$$

Pass to the limit as $j \rightarrow+\infty$ :

$$
W\left(t^{\prime}, x^{\sharp}\left(t^{\prime}\right)\right) \leq W\left(t^{\prime \prime}, x^{\sharp}\left(t^{\prime \prime}\right)\right)+\int_{t^{\prime}}^{t^{\prime \prime}} L\left(s, x^{\sharp}(s), \omega\right) d s .
$$

First consider the case $\left\{\left(t, x^{\sharp}(t)\right): t \in\left[t_{0}, T\right]\right\} \subseteq Q$. Summing (4.9) over each interval on which $u^{\sharp}$ is constant we have

$$
W\left(t_{0}, x^{\sharp}\left(t_{0}\right)\right) \leq W\left(T, x^{\sharp}(T)\right)+\int_{t_{0}}^{T} L\left(s, x^{\sharp}(s), u^{\sharp}(s)\right) d s .
$$

Now, $x^{\sharp}(T)=x^{*}(T)$ by defi nition and so, using 4.7)-4.3)-(4.4)

$$
\begin{aligned}
W\left(t_{0}, x^{\sharp}\left(t_{0}\right)\right) \leq & W\left(T, x^{*}(T)\right)+\int_{t_{0}}^{T} L\left(s, x^{\sharp}(s), u^{\sharp}(s)\right) d s \\
\leq & \psi\left(T, x^{*}(T)\right)+\int_{t_{0}}^{T} L\left(s, x^{\sharp}(s), u^{\sharp}(s)\right) d s \\
\leq & V\left(t_{0}, x_{0}\right)+\frac{\varepsilon}{2}-\int_{t_{0}}^{T} L\left(s, x^{*}(s), u^{*}(s)\right) d s \\
& +\int_{t_{0}}^{T} L\left(s, x^{\sharp}(s), u^{\sharp}(s)\right) d s \\
\leq & V\left(t_{0}, x_{0}\right)+\varepsilon<W\left(t_{0}, x^{\sharp}\left(t_{0}\right)\right) .
\end{aligned}
$$


This is a contradiction.

Suppose now $\left\{\left(t, x^{\sharp}(t)\right): t \in\left[t_{0}, T\right]\right\} \nsubseteq Q$. Defi ne

$$
\hat{\tau}:=\inf \left\{t \leq T:\left(s, x^{\sharp}(s)\right) \in Q \quad \forall s \in[t, T]\right\} .
$$

In particular $\left(\hat{\tau}, x^{\sharp}(\hat{\tau})\right) \in \partial Q$. Using the same argument to pass from (4.9) to 4.10), we obtain that for every $\tau>\hat{\tau}$

$$
W\left(\tau, x^{\sharp}(\tau)\right) \leq W\left(T, x^{*}(T)\right)+\int_{\tau}^{T} L\left(s, x^{\sharp}(s), u^{\sharp}(s)\right) d s
$$

and so

$$
\begin{aligned}
W\left(\tau, x^{\sharp}(\tau)\right) \leq & \psi\left(T, x^{*}(T)\right)+\int_{\tau}^{T} L\left(s, x^{\sharp}(s), u^{\sharp}(s)\right) d s \\
\leq & V\left(t_{0}, x_{0}\right)+\frac{\varepsilon}{2}-\int_{t_{0}}^{T} L\left(s, x^{*}(s), u^{*}(s)\right) d s \\
& +\int_{\tau}^{T} L\left(s, x^{\sharp}(s), u^{\sharp}(s)\right) d s .
\end{aligned}
$$

Since $L \geq 0$, we obtain for all $\tau>\hat{\tau}$

$$
\begin{aligned}
W\left(\tau, x^{\sharp}(\tau)\right) & \leq V\left(t_{0}, x_{0}\right)+\varepsilon \\
& \leq M-\varepsilon \\
& <W\left(t_{0}, x_{0}\right)-\varepsilon .
\end{aligned}
$$

Passing to the liminf as $\tau \rightarrow \hat{\tau}$ and using the lower semicontinuity of $W$, we conclude

$$
W\left(\hat{\tau}, x^{\sharp}(\hat{\tau})\right)<W\left(t_{0}, x_{0}\right)-\varepsilon
$$

and so by iiii)

$$
W\left(t_{0}, x_{0}\right)<W\left(t_{0}, x_{0}\right)-\varepsilon
$$

which is a contradiction. This concludes the proof of the theorem.

The hypotheses of the positiveness of $L$ is quite optimal as the next example shows. However, it is suffi cient that the system could not stay for too long in a region where the Lagrangian is negative, so one can relax the assumption $v$ ) in this way.

Example 1. Consider the system $\dot{x}=u, U=[-1,1]$ and $u=L^{1}(\mathbb{R} ; U), \Omega=\mathbb{R}^{2}, S=\mathbb{R} \times\{0\}$, $Q=\mathbb{R} \times]-1,1\left[\right.$ with the Lagrangian $L(t, x, u)=u^{2}+x^{4}-6 x^{3}+7 x^{2}$ (see Figure $\square$ ) and $\psi \equiv 0$ on $S$. Since the Lagrangian is negative in a region where the system can stay for an arbitrary interval of times, clearly the value function for this problem is equal to $-\infty$. If $W \equiv C$ on $\bar{Q}$ with $C$ negative constant, then $W$ verifi es all the hypotheses of the Theorem \, but $v$ ). In fact i), ii), iii) are obvious, while iv) holds because $L$ is positive on $Q$ and $W$ is differentiable on $Q$. So there exist inf nitely many functions $W$ defi ned on $\bar{Q}$ verifying the hypotheses of Theorem $\square$, but $v)$, which are different from $V$. 


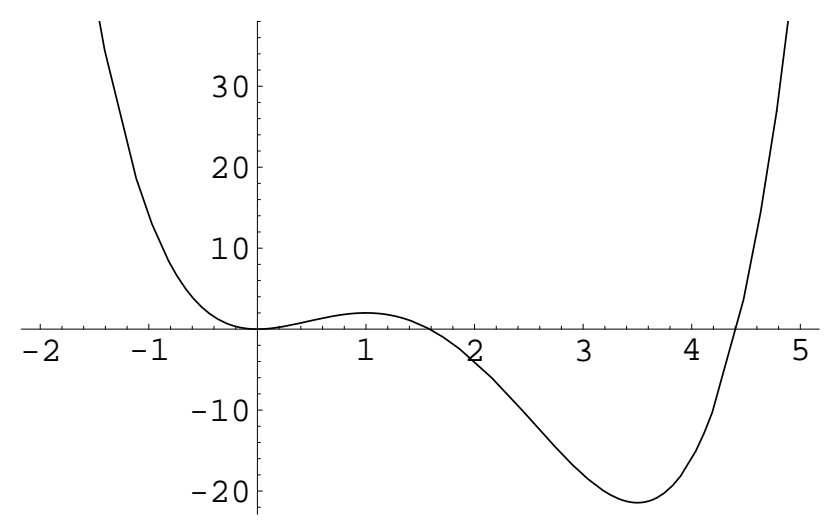

Figure 1: $L(t, x, 0)$

\section{Problem with infi nite time}

In this section we consider the control system (2.1) and assume that (A-11)-(A-5) hold with $0 \leq C_{R} \leq C$ for some $C>0$ and every $R>0$. Moreover we suppose that the target $S$ is a closed subset of $\mathbb{R} \times \mathbb{R}^{n}$ which satisfi es:

(*) For any $T>0$, there exists $(t, x) \in S$ with $t \geq T$.

Let $S_{1}$ be an open neighborhood of $S$ contained in $\Omega$. Assume that the fi nal cost $\psi$ is defi ned on $S_{1}$ and, if $d\left(\left(t, x\left(t ; u, t_{0}, x_{0}\right)\right), S\right) \rightarrow 0$ as $t \rightarrow+\infty$, then the trajectory $x\left(\cdot ; u, t_{0}, x_{0}\right)$ is defi nitively in $S_{1}$, that is:

(**) there exists $T>t$ such that $\left(s, x\left(s ; u, t_{0}, x_{0}\right)\right) \in S_{1}$ for all $s \geq T$.

Defi ne the value function $V\left(\hbar, x_{0}\right)$ as

$$
\inf _{\substack{u \in \mathcal{U} \\ d\left(\left(t, x\left(t ; t_{0}, t_{0}, x_{0}\right)\right), S\right) \rightarrow 0 \\ \text { as } t \rightarrow+\infty}}\left\{\int_{t_{0}}^{+\infty} L\left(s, x\left(s ; u, t_{0}, x_{0}\right), u(s)\right) d s+\limsup _{t \rightarrow+\infty} \psi\left(t, x\left(t ; u, t_{0}, x_{0}\right)\right)\right\}
$$

In other words, we consider only the trajectories that approach the target $S$ in infi nite time. Notice that this condition does not imply that $(T, x(T)) \notin S$ for every $T \geq t_{0}$.

We now prove a verifi cation theorem for a function $W$ defi ned on $\bar{Q}$, where $Q$ is an open subset of $\Omega$ containing the target.

Theorem 2. Let $Q \subseteq \Omega$ be an open subset containing $S$. Let $W: \bar{Q} \rightarrow \mathbb{R}$ be a lower semicontinuous function verifying the NDJ property along every time-varying vector fi eld $X$ of the type $f(t, x, u)$ with $u \in U$ fixed. Moreover assume that, for each $t, W(t, \cdot)$ is w.u.s.c. and that:

i) $W \geq V$.

ii) $W \leq \psi$ on $S_{1}$. 
iii) At every point $(t, x) \in \partial Q$ one has

$$
W(t, x)=\sup _{(s, y) \in Q} W(s, y) .
$$

iv) There exists a thin set $A \subseteq \Omega$ such that $W$ is differentiable in $Q \backslash A$ and satisfi es

$$
W_{s}(s, y)+\inf _{\omega \in U}\left\{W_{y}(s, y) \cdot f(s, y, \omega)+L(s, y, \omega)\right\} \geq 0 \quad \text { in } Q \backslash A .
$$

v) $L \geq 0$.

Then $W=V$ on $Q$. If $Q=\Omega$ we can drop hypotheses (iii) and Q .

The proof is eesentially similar to the proof of Theorem 1. For a complete proof of this theorem see also [8].

Remark 2. If we assume that there exists $\eta>0$ such that $S+B(0, \eta) \subseteq S_{1}$, where $B(0, \eta)$ is the ball in $\mathbb{R}^{n+1}$ centered in 0 with radius $\eta$, then hypothesis $(* *)$ obviously holds. In fact suppose $d\left(\left(t, x\left(t ; u, t_{0}, x_{0}\right)\right), S\right) \rightarrow 0$ as $t \rightarrow+\infty$. Then there exists $T>0$ such that $d\left(\left(s, x\left(s ; u, t_{0}, x_{0}\right)\right), S\right)<\frac{\eta}{2}$ for all $s \geq T$. So we can choose an element $(t(s), y(s)) \in S$ in order to have

$$
d\left(\left(s, x\left(s ; u, t_{0}, x_{0}\right)\right),(t(s), y(s))\right)<\frac{\eta}{2}
$$

for all $s \geq T$. So the points $\left(s, x\left(s ; u, t_{0}, x_{0}\right)\right) \in S+B(0, \eta) \subseteq S_{1}$ for every $s \geq T$.

Remark 3. We obtain a generalization of Theorems 1 and 1 considering the same problem (2.1) with assumptions $(A-7)-(A-4)$, but we accept at the same time all the trajectories that hit the target in fi nite time or that tend to the target in infi nite time. Obviously an analogous theorem a $7 l$ an $\mathbb{E}$ holds.

\section{References}

[1] M. Bardi, I. Capuzzo-Dolcetta, Optimal Control and Viscosity Solutions of Hamilton-Jacobi-Bellman Equations, Birkhäuser Boston Inc., Boston 1997.

[2] A. Bressan, Hyperbolic systems of conservation laws. The one-dimensional Cauchy problem, Oxford University Press, Oxford 2000.

[3] H. Brezis, Analyse fonctionnelle: Théorie et applications, Masson, Paris 1983.

[4] P. Cannarsa, A. Mennucci, C. Sinestrari, Regularity results for Solutions of a Class of Hamilton-Jacobi Equations, Arch. Rational Mech. Anal., 140 (1997), pp. 197-223.

[5] L. C. Evans, R. F. Gariepy, Measure Theory and Fine Properties of Functions, Studies in Advanced Mathematics, CRC press 1992.

[6] G. B. Folland, Real Analysis: Modern Techniques and their Applications, J. Wiley and sons, New York 1999.

[7] I. Fonseca, W. Gangbo, Degree theory in analysis and applications, Clarendon Press, Oxford, 1995. 
[8] M. Garavello, Verification theorems for Hamilton-Jacobi-Bellman equations, SIAM J. Control Optim. 42, (2003), no. 5, pp. 1623-1642.

[9] P. Hartman, Ordinary Differential Equations, John Wiley \& Sons Inc., New York 1964.

[10] M. Malisoff, On the Bellman equation for control problems with exit times and unbounded cost functionals, in proceedings of the $38^{\text {th }}$ IEEE Conference on Decision and Control, Phoenix, Arizona USA, 1999.

[11] M. Malisoff, H. J. Sussmann, Further Results on the Bellman Equation for Optimal Control Problems with Exit Times and Nonnegative Instantaneous Costs, in proceedings of 39th IEEE Conference on Decision and Control, Sydney, Australia 2000.

[12] B. Piccoli, Infinite time regular synthesis, ESAIM, Control Optim. Calc. Var. 3, (1998), pp. 381-405.

[13] B. Piccoli, H. J. Sussmann, Regular Synthesis and Sufficient Conditions for Optimality, SIAM J. Control Optim., 39 (2000), pp. 359-410.

[14] P. Soravia, $\mathcal{H}_{\infty}$ Control of Nonlinear Systems: Differential Games and Viscosity Solutions, SIAM J. Control Optim., 34 (1996), pp. 1071-1097. 